\title{
RELEVAMIENTO SOBRE EL MANEJO DE AGROQUÍMICOS EN CHACRAS DE LA PERIFERIA DE LA CIUDAD DE CORRIENTES
}

\author{
CASTILLO, Alicia E.; SANTILLÁN, Rubén A. y SOSA LÓPEZ, Angela \\ Qca. Orgánica y Biológica FCA- UNNE. - Sgto Cabral 2131- Corrientes. Argentina.
}

\section{GENERALIDADES}

Los pesticidas son ampliamente usados en la agricultura para incrementar los rendimientos de esta actividad, mediante el control de plagas. Dichos agroquímicos conllevan a que si no se controlan dosis, tiempo de carencia, depósito, etc., pueden acarrear distintos problemas, tanto referentes a la salud humana como al ambiente (Castillo et al., 2005; www.senasa.com).

En orden de mantener y mejorar su productividad, es sabido que los productores realizan aplicaciones de pesticidas, ya sea por prevención o ante la aparición de alguna plaga. Esto se realiza, aún existiendo desconocimiento mayoritario, en cuanto a tipos, dosis y momentos de aplicación de los pesticidas. Esta falta de conocimiento agrava los problemas de sanidad de los productos obtenidos, ya que la residualidad de ellos, representa peligros potenciales en la calidad de vida (Castillo et al., 2005; Nunes et al., 2005, 2006). La creciente demanda de alimentos a nivel mundial obliga al continuo estudio y adopción de nuevos métodos y técnicas de producción (biotecnología, manejo integrado de plagas) para incrementar tanto la calidad como la cantidad de los mismos. Aún así, los productos fitosanitarios seguirán desempeñando un rol importante en la protección de los cultivos en las próximas décadas. Sin embargo, es necesario conocer y trabajar en los distintos aspectos que hacen al manejo racional de agroquímicos para evitar efectos directos e indirectos sobre el hombre y el ambiente. Son diversos los inconvenientes producidos por el mal uso de fitosanitarios en cultivos hortícolas y frutícolas: elección de productos no adecuados, subdosis o sobredosis, presencia de residuos en productos frescos, descarte inadecuado de envases, falta de mantenimiento y manejo inadecuado de los equipos de aplicación, contaminación personal del aplicador, entre otros.

La legislación argentina, siguiendo los lineamientos tanto de SENASA como del Código Alimentario Argentino, establece requisitos relacionados con la capacitación de quienes son los encargados de aplicar dichos productos y todo lo referente al proceso productivo en su totalidad. Además incluye recomendaciones para la higiene del medio donde se desarrolla la producción asociado al suelo y al agua, y prácticas de manejo que pueden producir contaminaciones: fitosanitarios, las instalaciones, etc. (SAGPYA. 2005).

\section{Panorama general de la producción hortícola}

La horticultura en la Argentina se caracteriza por su amplia distribución geográfica y por la diversidad de las especias productivas

- Superficie: 600.000 hectáreas

- Producción:10.000.000 toneladas

Fuente: SAGPYA 2005.

En nuestra región, los sistemas de producción, en especial de hortalizas, han aumentado notablemente en los últimos años en la provincia de Corrientes. Esta actividad adquiere importancia a partir del año 1969, producida por un constante incremento de los cultivos tempranos y de primicia como consecuencia de la realización de obras de infraestructura. Estas contribuyeron a mejorar el transporte de la producción y en consecuencia de la comercialización.

Ante la falta de estudios relevantes acerca de la presencia e identificación de compuestos orgánicos contaminantes provenientes de pesticidas, especialmente los usados para combatir plagas (insectos, hongos, etc.,). Es imperativo encarar acciones que ayuden a obtener un relevamiento cuali y cuantitativo de dichos pesticidas a fin de que dicha información sea reportada a los sectores productivos y consumidores, para que se tomen las medidas adecuadas según la situación encontrada. Se han publicado algunos trabajos relativos a esta problemática como por ejemplo: Flores, V. y Lancelle, H. 2002; Castillo et al., 2003; Castillo et al., 2005; Castillo et al., 2006; Castillo et al., 2007 a, b; Castillo et al., 2008.

\section{Producción hortícola de la provincia de Corrientes}

- Superficie: 15.000 hectáreas

- Producción: 240.000 toneladas

Fuente: MPTT, Guía para el exportador agrícola

La superficie cultivada por la gran mayoría de los productores frutihortícolas oscila entre 1 y $7 \mathrm{ha}$; la mayor parte de la producción tiene como destino el Mercado Central de la ciudad de Corrientes. Este cuenta con 129 puestos, de los cuales 69 son fijos y 
60 en playa libre. Los primeros son ocupados por comerciantes mayoristas y los segundos son destinados a los productores correntinos. Desde este punto la mercadería es derivada al Mercado Central de Buenos Aires. Ante el hallazgo de residuos de fitosanitarios en los diferentes análisis de calidad realizados sobre mercadería proveniente de dicho mercado, en la actualidad el SENASA ha incrementado el control de los mismos en los últimos años con muestreos al azar (Comunicación personal, MPTT 2009).

El objetivo del presente trabajo consistió en determinar de qué manera son usados los pesticidas en chacras frutihortícolas de la periferia de la Ciudad de Corrientes.

\section{TRABAJO DE CAMPO}

\section{Locación y elección de las chacras frutihortícolas}

Se llevó a cabo una selección de un total de 18 unidades de producción hortícola, las cuales fueron localizadas e indicadas por técnicos del Ministerio de la Producción.

Se eligieron tres zonas bien diferenciadas: alrededor del Barrio Molina Punta (Zona I), periferia del pueblo de Santa Ana (Zona II) y Av. Maipú al sur (Zona III). Las mismas presentaban cultivos con modalidad de "tendaleros" o a campo abierto.

\section{Toma de datos}

La toma de datos se efectuó por observaciones, cuestionarios y entrevistas a dueños o encargados de las explotaciones agrícolas.

La metodología de dicha toma de datos consistió en:

1) Explicar brevemente el objetivo de la misma, $y$ que las respuestas eran anónimas

2) Se registraron por escrito las respuestas a cuestionarios, así como también las observaciones y hechos particulares.

Los cuestionarios incluyeron preguntas generales sobre manejo de pesticidas (dosis de aplicación, momentos de la misma, almacenamiento de envases, visita de profesionales del agro, medidas de protección, etc.).

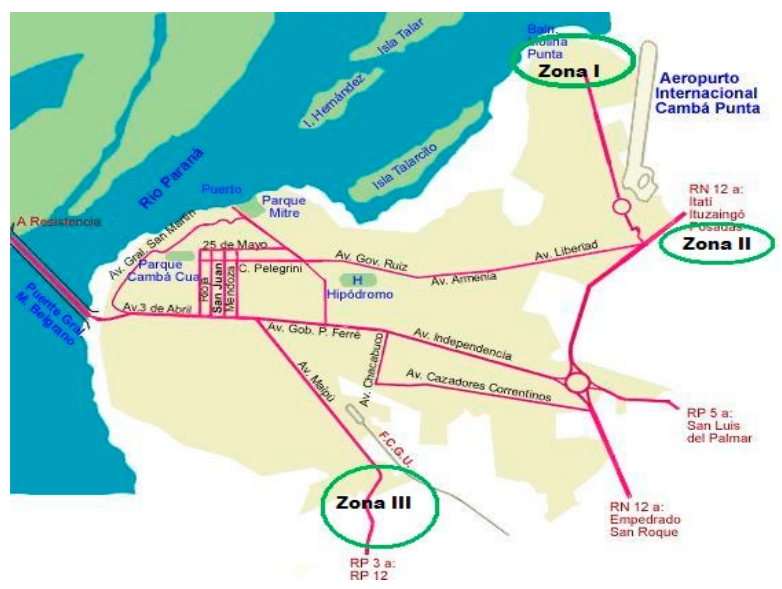

Cuadro 1. Distribución de la producción de acuerdo al sistema de cultivo

\begin{tabular}{lcc}
\hline & A campo abierto & Bajo cobertura \\
\hline Superficie en has. & 13.900 & 1.100 \\
Producción en Tn & 144.000 & 96.000 \\
\hline
\end{tabular}

Fuente: MPTT, Guía para el exportador agrícola

Las 18 unidades de producción incluidas en ese relevamiento se dedicaban al cultivo de lechuga, acelga, pimiento, perejil, zapallo, tomate y pimentón con fines de comercialización, bajo el diseño de tendaleros y/o campo abierto. En cada una de las visitas se pudieron observar las diferentes prácticas agrícolas que se utilizan, con grados diferenciados de tecnologías utilizadas, acorde a su alcance económico y también a su grado de responsabilidad en cuanto a las técnicas aplicadas.

\section{TRABAJO DE GABINETE}

- Confección de folleto informativo

- Procesamiento y evaluación de los datos relevados

- Elaboración de los Resultados

\section{RESULTADOS DE LA ENCUESTA/ ENTREVISTA}

Número de chacras visitadas: 18

Número de personas entrevistadas: 15

La diferencia entre chacras visitadas y entrevistadas, se debió a la negativa de productores de origen extranjero.

Cada una de las chacras visitadas fue identificada por un número, $\mathrm{y}$, los encuestados sometidos todos al mismo cuestionario.

A continuación se transcriben las respuestas a preguntas consideradas relevantes sobre el manejo de los agroquímicos.

Tiene visitas de personal técnico?

\section{Sí : $67 \%$ - No : $20 \%$ A veces : $13 \%$}

Se pudo observar que ese $67 \%$ de los productores que respondió positivamente, estaba asesorado por un Ingeniero Agrónomo y tenía información previa antes de comprar un fitosanitario Los profesionales pertenecían al Ministerio de la Producción. Generalmente las visitas se realizaban una vez por semana. Algunos productores formaban parte del Programa Agricultura Urbana y otros como el programa Yacarú Porá de La Secretaría de Desarrollo Humano de Corrientes. En cambio, en aquellos que no poseen visita de algún técnico, su comportamiento de la práctica agrícola, está basado fundamentalmente en su propia experiencia o por conocimientos heredados, y también por consejo de algún productor vecino.

Los agroquímicos a utilizar, en su totalidad, se trasladan en vehículos como automóvil, camioneta o 
motocicleta. Para el transporte no se utiliza ningún tipo de aislamiento, ni equipo adecuado, como extintores, material absorbente para derrames o recipiente con agua. A veces en contacto directo con la persona que lo transporta.

Realiza monitoreos de plagas, enfermedades y malezas?

Sí: 33\% - No: $67 \%$

Las aplicaciones de agroquímicos, en general eran de modo preventivo, sin tener en cuenta la presencia de la plaga, aunque en algunos casos eran de emergencia.

Lee las etiquetas y sus instrucciones antes de aplicar el fitosanitario?

\section{Sí : $33 \%$ - No : $47 \%$ - A veces : $20 \%$}

En las etiquetas se aconseja el uso de elementos de protección personal, pero sin tener criterios que permitan su correcta elección y utilización. Así hay elementos que son en general muy pocos usados entre los productores entrevistados como por ejemplo gafas, mascarillas y guantes. El no uso es más notable en épocas de primavera-verano, por la incomodidad que representa el equipo (dificultad en respiración, abundante transpiración, etc.). El factor económico también es crucial, hay productores de bajos recursos que deciden no adquirir ciertos elementos de seguridad debido a su costo. Por último se encuentra el factor cultural, siendo el modo de aplicación sin protección algo muy común entre los productores.

El $100 \%$ de los productores utilizaban mochila para la aplicación del fitoterápico.

En qué momento del día realiza la aplicación del agroquímico?

El $80 \%$ lo hace en horas de tarde, cuidando que ya no se encuentre el sol a pleno y no haya vientos.

\section{Que hace con el producto sobrante?}

La gran mayoría aplica toda la solución, hasta terminar el contenido de la mochila. Es necesario tener presente que en un envase de agroquímicos, luego de agotar su contenido y dejado un tiempo en reposo, se observa un residuo que representa hasta un 5\% del producto contenido. Este porcentaje depende de la viscosidad del mismo. Estos remanentes, si no son debidamente dispuestos, pueden transformarse en elementos potencialmente peligrosos para el sistema biótico.

\section{Que hace con los envases vacíos?}

Algunos productores los recolectan para quemarlos en alguna parte alejada de la chacra. Otros juntan los envases, realizan el triple enjuague aconsejado y los embolsan para luego ser recogidos por parte de un programa de recolección y disposición de los envases vacíos de los agroquímicos, instituido por el Mercado de Concentración y SENASA. Un solo productor respondió que volvía a utilizar los envases luego de que se terminara su contenido. Los envases que contuvieron productos fitosanitarios son legalmente considerados como Residuos Peligrosos según la Ley Nacional 24051. Esta circunstancia motiva que estos envases deban ser tratados de acuerdo a los requisitos que la norma legal impone.

\section{Tiene conocimientos de primeros auxilios?}

\section{Sí : $\mathbf{2 7 \%}$ No : $73 \%$}

La mayor parte de los productores encuestados no posee conocimientos de primeros auxilios en caso de intoxicación. Es importante que todo personal vinculado con las tareas agropecuarias conozca y pueda aplicar los primeros auxilios a un intoxicado, mientras se espera la llegada de un medico.

Conoce el tiempo de carencia de los productos que utiliza?

\section{Sí : $60 \%$ No : $40 \%$}

Se observa que del $60 \%$ que da un sí como respuesta, solamente unos pocos demostraban que en verdad tenía el conocimiento y sabía de lo que hablaba. Otra cuestión importante es que los productores tienen una idea errónea del tiempo de carencia, ya que ellos decían que aplicaban los productos los fines de semanas para poder llevar la cosecha los lunes al Mercado Central. La presencia de residuos depende del producto y su acción tóxica ya que cada uno de estos tiene un tiempo de degradación natural diferente, que varía en función del tipo de compuesto químico de que se trate. Por tal motivo es importante que el productor conozca el Tiempo de Carencia, que se define como: la cantidad de días que debe transcurrir entre la última aplicación de un producto fitosanitario y la cosecha o pastoreo de animales. En el caso de aplicaciones post cosecha se refiere al intervalo entre la última aplicación y el consumo del producto vegetal.

Conoce que son las buenas prácticas agrícolas?

La mayoría de los productores muestra interés en llevar adelante buenas prácticas, ya que son concientes cada vez más que los alimentos deben ser aptos para el consumo humano, garantizando de esta manera que se pueda acceder a diferentes mercados. Las buenas prácticas agrícolas se refieren a prácticas de manejo recomendadas para la producción vegetal desde la actividad primaria hasta el transporte y empaque que tienden asegurar la inocuidad y alcanzar una determinada calidad del producto. Hay una mayor predisposición a obtener información, y existe gran participación de los mismos a charlas y seminarios dados por el Ministerio de la Producción de la Ciudad de Corrientes, a través del Mercado de Concentración.

\section{Donde almacena los agroquímicos?}

En cada una de las chacras el modo y sitio de almacenaje de los plaguicidas fue diferente. En todas, la ubicación de dichos depósitos era peligrosa. Por ejemplo: heladera en desuso, utilizada para guardar 
los envases o piezas de material donde se guardan juntamente herramientas y productos fitosanitarios.

\section{CONCLUSIONES}

De acuerdo a los resultados que corresponden al cuestionario y a las observaciones personales, de este análisis cualitativo de la situación evaluada, surge que:

- La mayoría de los entrevistados manifestó conocer la peligrosidad del daño que pueden producir los fitosanitarios y la importancia del uso de los elementos de protección necesarios para evitar alguna contaminación y/o perjuicio, pero, a pesar de ello no actúan en consecuencia.

- Algunos productores y/o responsables de la aplicación de los agroquímicos manifestaron haber tenido en algún momento ciertas molestias (dolor de cabeza, mareos, etc.)

- La manipulación de plaguicidas se hace a partir de una capacitación informal, visualizando el trabajo de otros trabajadores no profesionales. Esta capacitación muchas veces incide en intoxicaciones si no se toman los recaudos necesarios. Por ello es de suma importancia el rol de los ingenieros agrónomos quienes al recorrer las quintas y supervisar los cultivos, recomiendan prácticas para el manejo de las plagas animales como vegetales, basadas en el control químico.

- Se puede decir que a pesar de que existe aún información insuficiente con respecto al manejo de fitosanitarios, que debe ser transmitida dentro del marco de productores hortícolas de nuestra región, son cada vez menos los productores que se muestran indiferentes sobre este tema y se interiorizan en el tema en cuestión.

- Este creciente interés se debería en parte a la información proporcionada por los técnicos visitantes incrementada en los últimos años, los controles de seguimiento por parte de las autoridades del Mercado de Concentración de Corrientes, de SENASA, y también por experiencia propia.

Con este trabajo se ha podido tener una visión, que si bien es acotada, sobre el uso de plaguicidas en nuestro cordón hortícola, sirve como paso inicial a otras investigaciones en este tema de vital importancia. Se sugiere ampliar el número de productores a encuestar, y completar la información con toma de muestras para el análisis de suelos, aguas y productos agropecuarios.

\section{Agradecimiento}

Se agradece a la Ing. Agr. Mariela Pletsch del Mercado de Concentración de la Ciudad de Corrientes, por su colaboración en aportar distintos datos para la ejecución de este trabajo.

\section{BIBLIOGRAFIA}

Castillo, A.E.; Rojas, J.M.; Monteros Solito, R.I., Narde1li, J.I., Guasch, G. 2003. Metodologías Usadas para la Determinación de Carbofuran (2,3- dihidro- 2,2dimetilbenzofuran-7-il metilcarbamato) en Muestras de Distinto Origen. AGROTECNIA, N 10: 15-20.

Castillo, A. E.; Subovsky, M.; Rodríguez, S.; Fernández, N.; Rojas, J. 2004. Efecto de la materia orgánica en la movilidad del carbamato en un Ultisol. XIX Congreso Argentino de la Ciencia del Suelo-Paraná Pp. 62

Castillo, A. E., Rodríguez, S. C., Subovsky, M. J. Fernández, N. 2005 Residualidad de un carbamato a distintas profundidades de columnas de suelos. AGROTECNIA N ${ }^{\circ}$ 14: 24-25

Castillo, A. E, Oggero, R. M., Subovsky, M. J Rodríguez, S. C. 2005. Residualidad de carbofuran (2,3 dihidro-2,2-dimetil-7-benzofuranil metilcarbamato) en Suelos Arenosos. Revista Científica Agropecuaria UNER.9 : 113-118

Castillo, A.E.; Subovsky, M.J..; Sosa-López, A.A.; Nunes, G.S. 2007 a. Persistencia de Carbofurán en un molisol con diferentes usos. Revista Científica UDO Agrícola 7: 228-237.

Castillo A. E.; Subovsky, M.J.; Sosa López, A.A. Nunes, G.S. 2007 b. Residuos de 2,3-dihidro-2,2-dimetilbenzofuran-7-il metilcarbamato (carbofuran en un suelo con y sin uso agrícola). Agrotecnia $\mathrm{N}^{\mathrm{o}} 17$ : 5-8.

Flores, V. A. Lancelle, H. G. 2002 El uso de plaguicidas en la producción hortícola en el área de influencia de la ciudad de Corrientes (Argentina) .CCyT. UNNE.

Nunes, G. S.; Oliveira Marques, C. V. V. C.; Castillo, A.; Badea, M.; Marty, J-L. 2005. Determinacao de residuos de insecticidas carbamatos em alimentos infantis utilizando biosensores amperométricos a base de enzimas acetilcolinesterases mutantes. Acta Toxicológica Argentina. 13: 2-8.

Nunes, G. S.; Oliveira Marques, C. V. V. C.; Castillo, A.; Badea, M.; Marty, J-L 2006. Anälise de residuos de insecticidas carbamatos en comidas infantis utilizando biossensores amperometricosa base de enzimas acetilcolinestases genéticamente modificadas. Analytica. 20: 56-67.

SAGPYA. Dirección Nacional de Alimentos. Buenas Prácticas Agrícolas. 2005. 75 pp. www.senasa.gov.ar. 